\title{
Jaundice Status
}

National Cancer Institute

\section{Source}

National Cancer Institute. Jaundice Status. NCI Thesaurus. Code C158584.

A clinical assessment or impression by direct observation indicating whether or not a patient has a condition of jaundice (is jaundiced). 